\title{
Fisioterapia na síndrome da fragilidade em idosos
}

\author{
Physical therapy in the fragility syndrome in elderly \\ Caroline Zanin ${ }^{1}$ \\ Matheus Santos Gomes Jorge² \\ Bruna Knob3 \\ Lia Mara Wibelinger ${ }^{4}$
}

\section{RESUMO}

O estudo buscou recrutar na literatura informações sobre a atuação da Fisioterapia na Síndrome de Fragilidade em Idosos. Foram selecionados manualmente artigos nas línguas portuguesa e inglesa indexados nas bases de dados eletrônicos SciELO, Lilcs e Pubmed partindo do descritor Idoso Fragilizado em cruzamento com as palavras-chaves: Fisioterapia e Envelhecimento todos de acordo com os descritores de Ciência da Saúde. Entre as medidas que podem ser realizadas para com o idoso frágil estão: a ação de uma equipe multidisciplinar, a realização do diagnóstico precoce, a utilização de ferramentas que permitam a identificação da fragilidade de forma rápida e a realização de atividades físicas.

\section{PALAVRAS-CHAVE:}

Idoso Fragilizado; Fisioterapia; Envelhecimento. 


\section{ABSTRACT}

This study sought to recruit informations in the literature about the acting of the Physical Therapy in the Fragility Syndrome in Elderly. Was manually selected articles in the portuguese and english language, indexed in the electronics database SciELO, LILACS and PubMed from the Fragility Elderly descriptors in a cross with the Physical Therapy and Aging keyword all of them according with the Health Science Descriptors. Between the measures that can be realized with the fragility elderly are the following: the action of a multidisciplinary group, the realization of the early diagnosis, the use of tools that allow the identification of the fragility in a fast way and the physical practice.

\section{KEYWORDS:}

Frail Elderly; Physical Therapy Speciality; Aging. 


\section{INTRODUÇÃO}

Envelhecer não é sinônimo de doenças crônicas e incapacitantes, mas o próprio processo fisiológico de envelhecimento produz alterações que favoreçam o aparecimento de algumas doenças nessa faixa etária (VALENÇA e SILVA, 2011). Este processo reúne alterações que, em conjunto com o aumento da prevalência de doenças crônicas, podem acarretar no aparecimento das síndromes geriátricas, dentre as quais a síndrome da fragilidade merece destaque (REIS et al., 2014).

Os idosos formam uma população heterogênea, a maior parte deles é ativa e capaz de realizar as atividades de vida diária sem qualquer dependência, ainda assim, uma pequena parcela é constituída de idosos frágeis, estes são os indivíduos que mais utilizam o sistema de saúde nessa faixa etária, ocupam a maior parte dos leitos e ainda os utilizam por mais tempo (CÂMARA; BASTOS e VOLPE, 2012).

A fragilidade é definida como uma síndrome médica com várias causas e fatores agregados, caracterizada pela diminuição da força, resistência e funções fisiológicas o que torna o indivíduo mais propenso para a dependência funcional ou até mesmo a morte. Na fragilidade o indivíduo está vulnerável ao desenvolvimento de dependência e mortalidade, além disso, ela pode ocorrer a partir de uma variedade de doenças e condições clínicas (MORLEY et al., 2013).

Essa síndrome em idosos caracteriza um problema de saúde pública e com o avançar da mesma, surge a necessidade de cuidados especiais, principalmente quando se trata de países em desenvolvimento, aonde grande parte da população possui baixa renda e para proceder cuidados no próprio domicílio (CORDEIRO et al., 2015).

Entre os fatores antecedentes físicos ou biológicos da fragilidade pode-se citar o declínio acumulativo em múltiplos sistemas orgânicos, alterações no peso corpóreo, desnutrição, ingestão nutricional inadequada, comorbidades, depressão, baixo nível de atividade física, déficit cognitivo, déficit sensorial e polifarmácia (ANDRADE et al., 2012).

Existe no Brasil uma carência de programas que visem a promoção, prevenção e recuperação da saúde dos idosos. $\mathrm{O}$ envelhecimento do idoso brasileiro se constitui por um aglomerado de deficiências nas atividades de vida diária, ao passo que ainda se está distante de atingir um envelhecimento próspero e saudável (ANDRADE e PEREIRA, 2009).

Sendo assim, este estudo buscou recrutar na literatura informações a respeito da Síndrome da Fragilidade em Idosos e a atuação da Fisioterapia.

\section{METODOLOGIA}

Este é um estudo do tipo revisão de literatura sistemática, na qual foram consultados manualmente artigos indexados nas bases de dados eletrônicos Scielo, Pubmed e Lilacs entre o período de janeiro de 2009 a janeiro de 2016. Foram selecionados artigos a partir do descritor Idoso Fragilizado (Frail Elderly) em cruzamento com as palavras-chaves Fisioterapia (Physical Therapy Speciality) e Envelhecimento (Aging) todos de acordo com os descritores de Ciência da Saúde (DeCS).

Incorporou-se ao estudo artigos do tipo revisão sistemática; ensaios clínicos e estudos de casos; artigos da língua portuguesa e da língua inglesa; artigos que caracterizassem o tema "Fisioterapia na Síndro-

Foram excluídos da pesquisa artigos que abordassem tratamento medicamentoso ou cirúrgico, artigos que abordassem outras patologias, artigos que abordassem outras síndromes e ainda artigos que não se enquadraram no tema proposto.

Após o recrutamento dos estudos e sua análise qualitativa os dados foram apresentados a seguir.

\section{RESULTADOS}

Realizou-se uma extensa pesquisa, onde foram encontrados 1.591 artigos. Foram lidos seus resumos e selecionados, inicialmente, 111 artigos potencialmente relevantes para o estudo, e, após uma análise qualitativa de forma criteriosa, finalmente, foram selecionados 30 artigos completos na íntegra que preencheram os critérios de inclusão, permitindo a fundamentação teórica da presente pesquisa. Dentre os artigos incluídos, 12 abordavam o tema Fisioterapia Na Síndrome da Fragilidade em Idosos dentro dos critérios exigidos. 
A figura 1 apresenta os resultados dos estudos recrutados e selecionados para o presente estudo.

Figura 1: Fluxograma da estratégia de seleção dos artigos.

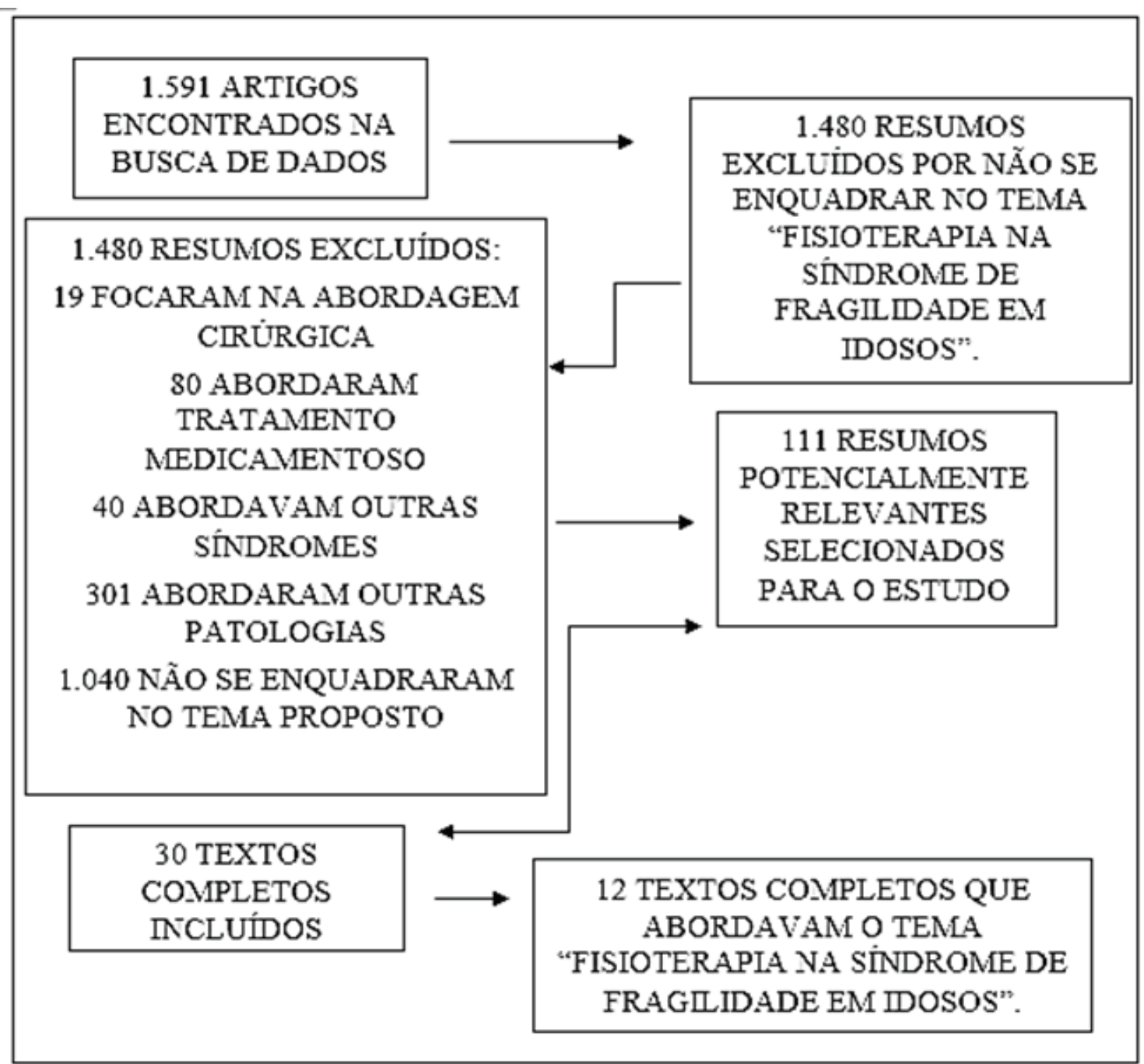


A tabela 1 apresenta características dos estudos recrutados sobre a força de preensão palmar em idosos.

Tabela 1: Caracterização dos estudos sobre a força de preensão palmar em idosos.

\begin{tabular}{|c|c|c|c|c|}
\hline Autores & Objetivo & Amostra & Protocolo & Desfecho \\
\hline $\begin{array}{l}\text { Tribess, VirtuA- } \\
\text { oso, de Olivei- } \\
\text { ra, } 2012^{8} \text {. }\end{array}$ & $\begin{array}{l}\text { Analisar a ativida- } \\
\text { de física em dife- } \\
\text { rentes domínios } \\
\text { como preditor de } \\
\text { ausência de fragili- } \\
\text { dade. }\end{array}$ & 622 indivíduos. & $\begin{array}{l}\text { A atividade física total e } \\
\text { seus domínios foram } \\
\text { avaliados pelo Questio- } \\
\text { nário Internacional de } \\
\text { Atividade Física. Foram } \\
\text { construídas curvas re- } \\
\text { ceiver operating charac- } \\
\text { teristic (ROC) e compa- } \\
\text { radas com a atividade } \\
\text { física em diferentes do- } \\
\text { mínios e ausência de fra- } \\
\text { gilidade. O índice de fra- } \\
\text { gilidade foi realizado } \\
\text { com base no estudo de } \\
\text { Fried. }\end{array}$ & $\begin{array}{l}\text { Praticar atividade física, } \\
\text { especialmente no tempo } \\
\text { de lazer ou acumulada em } \\
\text { diferentes domínios, con- } \\
\text { tribui para a prevenção da } \\
\text { fragilidade em idosos. }\end{array}$ \\
\hline $\begin{array}{l}\text { Costa Bertelli, } \\
\text { Liberalesso, } \\
2011^{9} .\end{array}$ & $\begin{array}{l}\text { Investigar relações } \\
\text { entre fragilidade e } \\
\text { medidas de ativi- } \\
\text { dade física. }\end{array}$ & 689 idosos. & $\begin{array}{l}\text { Foi utilizada versão } \\
\text { adaptada do Minnesota } \\
\text { Leisure Time Physical Ac- } \\
\text { tivity Questionnaire e es- } \\
\text { tabelecidos dois crité- } \\
\text { rios para as medidas de } \\
\text { atividade física: prática } \\
\text { regular de exercícios físi- } \\
\text { cos e taxa semanal de } \\
\text { gasto calórico em exer- } \\
\text { cícios físicos e em ativi- } \\
\text { dades domésticas. }\end{array}$ & $\begin{array}{l}\text { Os dados apontaram para } \\
\text { relações entre as variáveis } \\
\text { gênero, idade e renda fa- } \\
\text { miliar, de um lado, e os } \\
\text { indicadores de fragilidade } \\
\text { baixa força de preensão e } \\
\text { lentidão da marcha, de } \\
\text { outro; verificaram-se tam- } \\
\text { bém relação entre gênero } \\
\text { e doenças crônicas. }\end{array}$ \\
\hline $\begin{array}{l}\text { Lenardt, Sousa } \\
\text { Rocha, Carneiro } \\
\text { Hammerschmi- } \\
\text { dt Kolb, Betiolli } \\
\text { Elero, Ribeiro } \\
\text { Kohlbeck de } \\
\text { Melo Neu, } \\
2013^{10} .\end{array}$ & $\begin{array}{l}\text { Investigar a pré- } \\
\text {-fragilidade e os fa- } \\
\text { tores associados a } \\
\text { essa condição, } \\
\text { considerando o ní- } \\
\text { vel de atividade fí- } \\
\text { sica dos idosos. }\end{array}$ & 195 idosos. & $\begin{array}{l}\text { Os dados foram coleta- } \\
\text { dos mediante questio- } \\
\text { nário sociodemográfi- } \\
\text { co/clínico e nível de ati- } \\
\text { vidade física. }\end{array}$ & $\begin{array}{l}73 \text { idosos possuíam dimi- } \\
\text { nuição do nível de ativida- } \\
\text { de física, sendo classifica- } \\
\text { dos em condição de pré- } \\
\text {-fragilidade para esse } \\
\text { componente. }\end{array}$ \\
\hline
\end{tabular}




\begin{tabular}{|c|c|c|c|c|}
\hline Autores & Objetivo & Amostra & Protocolo & Desfecho \\
\hline $\begin{array}{l}\text { Câmara Caseri, } \\
\text { Bastos Corrêa, } \\
\text { Volpe Fernan- } \\
\text { des Tinoco, } \\
2012^{3} \text {. }\end{array}$ & $\begin{array}{l}\text { Averiguar os efei- } \\
\text { tos dos ER na fisio- } \\
\text { patologia da sín- } \\
\text { drome da fragili- } \\
\text { dade. }\end{array}$ & - & $\begin{array}{l}\text { Foi realizada uma revi- } \\
\text { são bibliográfica do pe- } \\
\text { ríodo de } 2004 \text { a } 2010, \\
\text { por meio das bases de } \\
\text { dados LILACS, MEDLINE } \\
\text { e PubMed. }\end{array}$ & $\begin{array}{l}\text { O ER deve ser indicado co- } \\
\text { mo opção terapêutica pa- } \\
\text { ra idosos frágeis ou pré- } \\
\text {-frágeis que não apresen- } \\
\text { tem contraindicações pa- } \\
\text { ra realização desta moda- } \\
\text { lidade de exercício. Os ER } \\
\text { atuam de forma sistêmica } \\
\text { na reversão ou minimiza- } \\
\text { ção dos efeitos da sarco- } \\
\text { penia exercendo influên- } \\
\text { cia positiva na síndrome } \\
\text { da fragilidade. }\end{array}$ \\
\hline $\begin{array}{l}\text { Lustosa, Silva, } \\
\text { Coelho, Pereira, } \\
\text { Parentoni, Pe- } \\
\text { reira, } 2011^{11} \text {. }\end{array}$ & $\begin{array}{l}\text { Verificar o efeito } \\
\text { do treinamento de } \\
\text { força muscular } \\
\text { com carga na ca- } \\
\text { pacidade funcional } \\
\text { e força muscular } \\
\text { dos extensores do } \\
\text { joelho e sua asso- } \\
\text { ciação, após trei- } \\
\text { namento, em ido- } \\
\text { sas pré-frágeis da } \\
\text { comunidade. }\end{array}$ & 32 idosas. & $\begin{array}{l}\text { Avaliou-se a capacidade } \\
\text { funcional (Timed Up and } \\
\text { Go - TUG e velocidade } \\
\text { de marcha - TC10) e a } \\
\text { força muscular dos ex- } \\
\text { tensores do joelho nas } \\
\text { velocidades angulares } \\
\text { de } 60 \text { e } 180 \% \text { s. Para o } \\
\text { fortalecimento muscu- } \\
\text { lar, utilizou-se carga de } \\
75 \% \text { de resistência má- } \\
\text { xima (1RM), durante dez } \\
\text { semanas, três vezes/se- } \\
\text { mana. }\end{array}$ & $\begin{array}{l}\text { O treinamento produziu } \\
\text { melhora da potência mus- } \\
\text { cular e capacidade funcio- } \\
\text { nal. A melhora da potên- } \\
\text { cia associou-se à melhora } \\
\text { funcional, importante va- } \\
\text { riável para a qualidade de } \\
\text { vida de idosas pré-frágeis. }\end{array}$ \\
\hline $\begin{array}{l}\text { Geraedts,, Zijls- } \\
\text { tra, Z Z hang, } \\
\text { Bulstra, Ste- } \\
\text { vens, } 2014^{12} \text {. }\end{array}$ & $\begin{array}{l}\text { Avaliar a adesão e } \\
\text { eficácia de um pro- } \\
\text { grama de ativida- } \\
\text { de física para ido- } \\
\text { sos dependentes } \\
\text { conduzidos pelo } \\
\text { monitoramento da } \\
\text { mobilidade através } \\
\text { de um sensor de } \\
\text { atividade física e } \\
\text { feedback remoto } \\
\text { usando um tablet. }\end{array}$ & $\begin{array}{l}50 \text { idosos frá- } \\
\text { geis. }\end{array}$ & $\begin{array}{l}\text { A amostra foi submeti- } \\
\text { da a } 6 \text { meses de exercí- } \\
\text { cios fornecidos em for- } \\
\text { ma de vídeo e monitora- } \\
\text { dos por um sensor. Os } \\
\text { participantes se exerci- } \\
\text { taram } 5 \text { vezes por sema- } \\
\text { na. Os exercícios foram } \\
\text { edificados em níveis e } \\
\text { adaptados individual- } \\
\text { mente. }\end{array}$ & $\begin{array}{l}\text { O programa de atividade } \\
\text { física orientado pela mo- } \\
\text { nitorização da mobilidade } \\
\text { através de um sensor e } \\
\text { instruções usando um ta- } \\
\text { blet é um método inova- } \\
\text { dor para a estimulação da } \\
\text { atividade física em idosos } \\
\text { frágeis. Se os participan- } \\
\text { tes seguem o programa, o } \\
\text { mesmo irá resultar em } \\
\text { maior atividade física diá- } \\
\text { ria, maior força e equilí- } \\
\text { brio avaliada por testes } \\
\text { físicos em comparação à } \\
\text { linha de base. }\end{array}$ \\
\hline
\end{tabular}




\begin{tabular}{|c|c|c|c|c|}
\hline Autores & Objetivo & Amostra & Protocolo & Desfecho \\
\hline $\begin{array}{l}\text { Cadore, Mo- } \\
\text { neo, Mensat, } \\
\text { Muñoz, Casas- } \\
\text {-Herrer, Rodri- } \\
\text { guez-Mañas, Iz- } \\
\text { q u i e r d o, } \\
2014^{13} \text {. }\end{array}$ & $\begin{array}{l}\text { Investigar os efei- } \\
\text { tos de uma inter- } \\
\text { venção multicom- } \\
\text { ponente de exercí- } \\
\text { cio sobre a força } \\
\text { muscular, a inci- } \\
\text { dência de quedas e } \\
\text { os resultados fun- } \\
\text { cionais em pacien- } \\
\text { tes idosos frágeis } \\
\text { que sofrem de de- } \\
\text { mência após con- } \\
\text { tenção física a lon- } \\
\text { go prazo, seguido } \\
\text { por } 24 \text { semanas de } \\
\text { interrupção de } \\
\text { treinamento. }\end{array}$ & $\begin{array}{l}18 \text { pacientes } \\
\text { idosos frágeis } \\
\text { com demência } \\
\text { leve. }\end{array}$ & $\begin{array}{l}\text { Realizou-se um progra- } \\
\text { ma de exercícios com } \\
\text { múltiplos componentes, } \\
\text { que consistiu de } 4 \text { sema- } \\
\text { nas de caminhada, equi- } \\
\text { líbrio e exercícios cogni- } \\
\text { tivos, seguido por } 4 \text { se- } \\
\text { manas de exercícios de } \\
\text { resistência realizados } \\
\text { duas vezes por semana } \\
\text { [8-12 repetições em } 20 \\
50 \% \text { da de uma repeti- } \\
\text { ção máxima ( } 1 \text { RM)], } \\
\text { combinada com a cami- } \\
\text { nhada, equilíbrio e exer- } \\
\text { cícios cognitivos. }\end{array}$ & $\begin{array}{l}\text { A intervenção com exercí- } \\
\text { cios multicomponentes } \\
\text { produziu melhora na for- } \\
\text { ça muscular, capacidade } \\
\text { de equilíbrio e marcha e } \\
\text { diminuiu a incidência de } \\
\text { quedas em pacientes ido- } \\
\text { sos frágeis com demência } \\
\text { após longo período de } \\
\text { contenção física. }\end{array}$ \\
\hline $\begin{array}{l}\text { Cadore, Casas- } \\
\text {-Herrer, Zam- } \\
\text { bom-Ferraresi, } \\
\text { Idoate, Millor, } \\
\text { Gómez, Izquier- } \\
\text { do, } 2014^{14} \text {. }\end{array}$ & $\begin{array}{l}\text { Examinar os efei- } \\
\text { tos do treinamen- } \\
\text { to com múltiplos } \\
\text { componentes em } \\
\text { potência muscular, } \\
\text { massa muscular, e } \\
\text { atenuação tecido } \\
\text { muscular; o risco } \\
\text { de quedas; e resul- } \\
\text { tados funcionais } \\
\text { em nonagenários } \\
\text { frágeis. }\end{array}$ & $\begin{array}{l}24 \text { idosos (91,9 } \\
\pm 4,1 \text { anos) fo- } \\
\text { ram randomi- } \\
\text { zados para Gl e } \\
\text { GC. }\end{array}$ & $\begin{array}{l}\text { O GI realizou um pro- } \\
\text { grama de } 12 \text { semanas } \\
\text { duas vezes por semana, } \\
\text { exercício composto por } \\
\text { treinamento de força } \\
\text { muscular ( } 8-10 \text { repeti- } \\
\text { ções, } 40-60 \% \text { da de uma } \\
\text { repetição máxima), } \\
\text { combinada com equilí- } \\
\text { brio e marcha. }\end{array}$ & $\begin{array}{l}\text { A intervenção do exercício } \\
\text { multicomponente utiliza- } \\
\text { do no presente estudo re- } \\
\text { sultou em melhorias na } \\
\text { força e desempenho de } \\
\text { potência, hipertrofia mus- } \\
\text { cular, infiltração de gor- } \\
\text { dura intramuscular, e os } \\
\text { resultados funcionais e re- } \\
\text { duziu a incidência de que- } \\
\text { das em nonagenários frá- } \\
\text { geis institucionalizados. }\end{array}$ \\
\hline $\begin{array}{l}\text { Cadore, Rodrí- } \\
\text { guez-Mañas, } \\
\text { Sinclair, Izquier- } \\
\text { do, } 2013^{15} \text {. }\end{array}$ & $\begin{array}{l}\text { Recomendar estra- } \\
\text { tégias que melho- } \\
\text { rem a capacidade } \\
\text { funcional em idosos } \\
\text { frágeis com base na } \\
\text { literatura científica, } \\
\text { incidindo especial- } \\
\text { mente em progra- } \\
\text { mas de exercício su- } \\
\text { pervisionado que } \\
\text { melhoraram a força } \\
\text { muscular, risco de } \\
\text { queda, equilíbrio e } \\
\text { capacidade de mar- } \\
\text { cha. }\end{array}$ & - & $\begin{array}{l}\text { Scielo, Science Citation } \\
\text { Index, MEDLINE, bases } \\
\text { de dados Scopus, Des- } \\
\text { porto Discus, e Science- } \\
\text { Direct foram pesquisa- } \\
\text { das no período de } 1990 \\
\text { a } 2012 \text {. }\end{array}$ & $\begin{array}{l}\text { Um programa de exercício } \\
\text { que consiste de força, re- } \\
\text { sistência e treinamento de } \\
\text { equilíbrio parece ser a me- } \\
\text { Ihor estratégia para me- } \\
\text { Ihorar a marcha, equilí- } \\
\text { brio e força, bem como } \\
\text { reduzir a taxa de quedas } \\
\text { em idosos e, consequen- } \\
\text { temente, a manutenção } \\
\text { da sua capacidade funcio- } \\
\text { nal durante o envelheci- } \\
\text { mento. }\end{array}$ \\
\hline
\end{tabular}




\begin{tabular}{|c|c|c|c|c|}
\hline Autores & Objetivo & Amostra & Protocolo & Desfecho \\
\hline $\begin{array}{l}\text { Fairhall, Sher- } \\
\text { rington, Kurrle, } \\
\text { L o r d, L o - } \\
\text { ckwood, Came- } \\
\text { ron, } 2012^{16} \text {. }\end{array}$ & $\begin{array}{l}\text { Avaliar o impacto } \\
\text { de uma interven- } \\
\text { ção multifatorial } \\
\text { na incapacidade } \\
\text { relacionada à mo- } \\
\text { bilidade em idosos } \\
\text { frágeis. }\end{array}$ & $\begin{array}{l}241 \text { idosos frá- } \\
\text { geis. }\end{array}$ & $\begin{array}{l}\text { O GE recebeu, uma in- } \\
\text { tervenção interdiscipli- } \\
\text { nar de } 12 \text { meses. Dois } \\
\text { fisioterapeutas elabora- } \\
\text { ram um programa de } \\
\text { exercícios em casa visan- } \\
\text { do mobilidade e gestão } \\
\text { coordenada das condi- } \\
\text { ções psicológicas e mé- } \\
\text { dicas com outros profis- } \\
\text { sionais de saúde. O GC } \\
\text { recebeu cuidados habi- } \\
\text { tuais. }\end{array}$ & $\begin{array}{l}\text { A intervenção reduziu in- } \\
\text { capacidade relacionada à } \\
\text { mobilidade em idosos frá- } \\
\text { geis. O benefício foi evi- } \\
\text { dente em ambos os níveis } \\
\text { de participação e de ativi- } \\
\text { dades de incapacidade re- } \\
\text { lacionada à mobilidade. }\end{array}$ \\
\hline $\begin{array}{l}\text { Villareal, Smith, } \\
\text { Sinacore, Shah, } \\
\text { Mittendorfer, } \\
2011^{17} \text {. }\end{array}$ & $\begin{array}{l}\text { Determinar o efei- } \\
\text { to de um PTF de } 3 \\
\text { meses de duração } \\
\text { na resistência, for- } \\
\text { ça muscular, massa } \\
\text { muscular e na taxa } \\
\text { de síntese de pro- } \\
\text { teína muscular em } \\
\text { idosos moderada- } \\
\text { mente frágeis e } \\
\text { obesos. }\end{array}$ & $\begin{array}{l}9 \text { idosos (65-80 } \\
\text { anos de idade). }\end{array}$ & $\begin{array}{l}\text { Cada sujeito completou } \\
\text { um programa de exercí- } \\
\text { cios por } 3 \text { meses; com- } \\
\text { posição corporal, fun- } \\
\text { ção física, resistência, e } \\
\text { as taxas de síntese pro- } \\
\text { têica muscular esquelé- } \\
\text { tica durante basal, as } \\
\text { condições pós-absortivo } \\
\text { e alimentação foram } \\
\text { avaliados antes e no fi- } \\
\text { nal do período de trei- } \\
\text { namento. }\end{array}$ & $\begin{array}{l}\text { Um programa de treina- } \\
\text { mento físico tem efeitos } \\
\text { benéficos sobre a massa } \\
\text { muscular e a função física } \\
\text { e deve, portanto, ser reco- } \\
\text { mendada para frágeis, } \\
\text { idosos obesos. }\end{array}$ \\
\hline $\begin{array}{l}\text { Zak, Swine, } \\
\text { Grodzicki, } \\
2009^{18} \text {. }\end{array}$ & $\begin{array}{l}\text { Determinar se um } \\
\text { programa de exer- } \\
\text { cício intensivo, } \\
\text { combinado com } \\
\text { suplementação } \\
\text { nutricional, pode } \\
\text { melhorar e ajudar } \\
\text { a manter a força } \\
\text { muscular individu- } \\
\text { al e mobilidade, e, } \\
\text { melhorar a capaci- } \\
\text { dade funcional in- } \\
\text { dividual. }\end{array}$ & $\begin{array}{l}91 \text { idosos frá- } \\
\text { geis (F } 71 \text { M 20; } \\
\text { com idade mé- } \\
\text { dia de } 79 \text { anos) } \\
\text { divididos em } \\
\text { quatro grupos. }\end{array}$ & $\begin{array}{l}\text { G1 realizou ERP + EOF } \\
+ \text { SN. G2 realizou ERP + } \\
\text { EOF + placebo; G3 rea- } \\
\text { lizou EP + EOF + SN. G4 } \\
\text { - SE + EOF + placebo. } \\
\text { Cada sessão durou } 45 \\
\text { min. e foi realizada } 5 \text { ve- } \\
\text { zes por semana. }\end{array}$ & $\begin{array}{l}\text { Diferenças significativas } \\
\text { na força muscular foram } \\
\text { observados tanto no G1 e } \\
\text { G2. Melhorias notáveis } \\
\text { em mobilidade individual } \\
\text { foram relatados no G3 e } \\
\text { G4. O programa realizado } \\
\text { demonstrou potencial } \\
\text { significativo para melhora } \\
\text { do estado geral funcional } \\
\text { em idosos frágeis em ter- } \\
\text { mos da capacidade de ca- } \\
\text { minhar individual e força } \\
\text { muscular. }\end{array}$ \\
\hline
\end{tabular}

ER: exercícios resistidos. Gl: grupo intervenção. GC: grupo controle. GE: grupo experimental. PTF: programa de treinamento físico. F: feminino. M: masculino. G1: grupo um. G2: grupo dois. G3: grupo três. G4: grupo quatro. ERP: exercícios de resistência progressiva. EOF: exercícios orientados funcionalmente. SN: suplementação nutricional. EP: exercícios padrão. SE: exercícios Standard. Min: minutos. 
Entre estudos reunidos que caracterizam o tema Fisioterapia na Síndrome da Fragilidade em Idosos dois exploraram os exercícios resistidos (CÂMARA; BASTOS e VOLPE, 2012) e (LUSTOSA et al., 2011), quatro estudos analisaram os efeitos da atividade física (TRIBESS; VIRTUOSO e OLIVEIRA, 2012), (COSTA e NERI, 2011), (LENARDT et al., 2013), (GERAEDTS et al., 2014) e outros seis estudos investigaram a aplicação de exercícios variados (CADORE et al., 2014), (CADORE et al., 2014), (CADORE et al., 2013), (FAIRHALL et al., 2012), (VILLAREAL et al., 2011) e (ZOK; SWINE e GRODZICKI, 2009). Um estudo concluiu que a atividade física contribui para prevenir a fragilidade em idosos (TRIBESS; VIRTUOSO e OLIVEIRA, 2012). Um estudo de Costa e Neri (2011) investigou as relações entre fragilidade e medidas de atividade física e os dados encontrados relacionam as variáveis gênero, idade e renda familiar e os indicadores de fragilidade foram apontados como baixa força de preensão palmar e lentidão na marcha, a relação entre gênero e doenças crônicas também foi apontada. Outra pesquisa buscou identificar a pré fragilidade e os fatores associados a ela segundo os níveis de atividade física e concluiu que de 195 idosos, 73 deles possuíam diminuição da atividade física e foram classificados como pré frágeis (LENARDT et al., 2013). Uma investigação de Câmara, Bastos e Volpe (2012) demonstrou os efeitos positivos dos exercícios resistidos em idosos frágeis ou pré frágeis, salientando que os mesmos atuam na redução da sarcopenia. Uma análise averiguou os efeitos do treinamento muscular com carga na capacidade funcional de uma musculatura específica em idosas pré frágeis e concluiu que o treinamento produziu melhora na potência, força e consequentemente na qualidade de vida das idosas (LUSTOSA et al., 2011). Um estudo propôs um programa de atividade física orientado por um tablet e um sensor e concluiu que o mesmo pode resultar em maior atividade física diária e maior força e equilíbrio avaliada por testes físicos em comparação à linha de base (GERAEDTS et al., 2014). Outras três pesquisas comprovaram que intervenção sistemática por exercícios multicomponentes produziu vários benefícios, tais como melhora na força muscular, capacidade de equilíbrio e marcha e diminuiu a incidência de quedas em pacientes idosos frágeis com demência após lon- go período de contenção física (CADORE et al., 2014), além disso resultou em melhorias na força e desempenho de potência, hipertrofia muscular e infiltração de gordura intramuscular (CADORE et al., 2014) e a manutenção da sua capacidade funcional durante envelhecimento (CADORE et al., 2013). Outro estudo verificou que um programa de intervenção multifatorial reduziu incapacidade relacionada à mobilidade em idosos frágeis (FAIRHALL et al., 2012), outra pesquisa ainda constatou que um programa de treinamento físico tem efeitos benéficos sobre a massa muscular e a função física e deve, portanto, ser recomendada para frágeis, idosos obesos (VILLAREAL et al., 2011) e um estudo verificou que um programa de exercício intensivo combinado com suplementação nutricional demonstrou potencial significativo para melhora do estado geral funcional em idosos frágeis em termos da capacidade de caminhar individual e força muscular (ZOK; SWINE e GRODZICKI, 2009). A amostra dos estudos incluídos variou de 09 para 689 indivíduos idosos.

\section{DISCUSSÃO}

A fragilidade pode ser definida como o envelhecimento malsucedido, sendo ele o produto de todas as situações vividas pelo indivíduo em toda sua vida. Essas situações podem ser afetadas por diversos fatores, tais como fatores biológicos, psicológicos e sociais (FERNANDES; ANDRADE e NOBREGA, 2010).

$O$ envelhecimento constitui uma preocupação e uma realidade mundial. Há uma transição do perfil demográfico da população, gerando assim um aumento brusco do número de idosos frágeis frente à necessidade de institucionalização (CORDEIRO et al., 2015). A longevidade está associada a fragilização, isso torna o idoso vulnerável às diversas situações de vida e saúde. No Brasil, pelo menos $85 \%$ dos idosos apresentem uma doença crônica ou mais, e destes, $10 \%$ com sobreposição de afecções concomitantes. Assim, a situação de cronicidade e longevidade atual dos brasileiros contribuem para o aumento de idosos com limitações funcionais que necessitam de cuidados constantes (GONÇALVES et al., 2006).

A fragilidade é uma síndroma multidimensional caracterizada pela perda de reserva física e cognitiva 
o que leva o indivíduo ao acúmulo de incapacidades e maior vulnerabilidade a outras diversas situações, ela está relacionada, principalmente, com a idade e se estende além do estado de incapacidade funcional e de doença mórbida (MCDERMID; STELFOX e BAGSHAW, 2011). A síndrome é mais presente em idades avançadas e se relaciona com diversos tipos de composição corporal, além disso esses indicadores também estão relacionados com maior risco de doenças crônicas, como a diabetes, hipertensão arterial e doenças cardiovasculares (MORETTO et al, 2012).

A incapacidade funcional em idosos se tornou um grande problema de saúde pública, causando inúmeros impactos na vida do indivíduo, seus familiares e nos serviços de saúde. Compete aos profissionais de saúde a identificação precoce da disfunção e fatores relacionados a ela, por isso a avaliação global do indivíduo deve ser incorporada como rotina aos serviços de saúde em todos os níveis de atenção. A associação entre incapacidade funcional, sexo e grupo etário demonstram que ações em saúde devem ser dirigidas principalmente às mulheres idosas e aos idosos longevos que são potencialmente suscetíveis a desenvolver incapacidades (BRITO; MENEZES e OLINDA, 2015).

Um estudo de Pegorari e Tavares (2014) demonstrou algumas situações que podem associar-se a fragilidade ou pré fragilidade: a ausência de companheiro está associada à pré fragilidade, enquanto que a hospitalização, incapacidade funcional para atividades básicas de vida diária e indicativo de depressão associaram-se à fragilidade. Outro estudo de Lana e Schneider (2014) verificou que idosos de maior idade, com menor escolaridade, portadores de doenças crônicas prévias, institucionalizados, em uso crônico de medicamentos, diminuição da altura e poucas relações sociais estão mais presentes entre os idosos frágeis. A redução das atividades físicas também é um indicador de fragilidade e a existência da mesma do fenótipo sugere uma predisposição a síndrome, por isso é importante a prática de atividade física regular, ela evita a perda da mobilidade e auxilia a manter a independência e a autonomia do idoso (LENARDT et al., 2013).

É importante detectar a fragilidade de forma precoce, isso reduz o risco de morbidade, mortalidade e hospitalização de idosos, dessa forma é relevante quesejarealizadaumaavaliaçãoclínicamultidisciplinar de forma periódica no idoso. É necessário também o uso de ferramentas simples que permitam a identificação do risco e/ou de fragilidade, isso poderá propiciar o desenvolvimento de práticas preventivas e intervenções apropriadas (STORTI et al., 2013).

Para a conservação da saúde da população idosa é fundamental a avaliação constante e o acompanhamento nutricional desses indivíduos na vivência clínica. A identificação precoce de déficits relacionados ao estado nutricional dos idosos permite a elaboração de protocolos e planejamentos individualizados para atuar como forma de prevenção para a síndrome da fragilidade (MORETTO et al., 2012).

Independente das condições de saúde dos indivíduos o envelhecimento requer cuidados especiais dos sistemas sociais e de saúde. $\mathrm{O}$ atendimento domiciliar ao idoso frágil é uma prática crescente e permite ao profissional de saúde estabelecer, junto aos familiares, condutas mais adequadas diante das necessidades da pessoa idosa (VALENÇA e SILVA, 2011).

Entre as principais estratégias de cuidado a saúde do idoso está a sensibilização dos profissionais da saúde que interagem com os idosos, seus cuidadores e familiares. Essa conduta auxilia na identificação de fatores de risco para a fragilidade do idoso, como também auxilia para o desenvolvimento de medidas de prevenção com o objetivo de evitar ou diminuir a ocorrência de situações que possam interferir de forma negativa na saúde do idoso. Além disso, no cuidado ao idoso frágil ou pré frágil a ação de uma equipe interdisciplinar é de grande valia, isso objetiva atuar não somente na reabilitação, mas na promoção, prevenção e educação do paciente para contribuir para a melhora global do indivíduo (REMOR; BÓS e WERLONG, 2011).

É importante o diagnóstico correto e o conhecimento do perfil do idoso hospitalizado, isso permite mais aprofundamento relacionado aos cuidados interdisciplinares com o indivíduo hospitalizado. Uma das barreiras para o melhor entendimento do declínio funcional desses pacientes é a falta de ferramentas e critérios padronizados para avaliar a síndrome da fragilidade em pessoas idosas (OLIVEIRA et al., 2013). Há necessidade de uma pa- 
dronização conceitual, visando elaborar condutas preventivas e de promoção da saúde em idosos portadores da síndrome de fragilidade, visto que a mesma se caracteriza por um déficit complexo que demanda de ações isoladas para prevenir, retardar ou impedir sua evolução junto ao processo de envelhecimento (LANA e SCHNEIDER, 2014).

A atividade física produz benefícios significativos na qualidade de vida e funcionalidade da população idosa. $O$ treinamento resistido realizado de forma regular e contínua pode reverter e/ou minimizar os efeitos da sarcopenia, que é considerada uma das principais causas de fragilidade em idosos (CÂMARA; BASTOS e VOLPE, 2012).

O exercício resistido propõe uma série de benefícios, entre eles o aumento de alguns hormônios sexuais responsáveis pela manutenção do sistema musculoesquelético. Enquanto o exercício físico exaustivo pode suprimir a atuação do sistema imune, os exercícios moderados estimulam o sistema imune e podem ser responsáveis pela relação dos exercícios e a redução de doenças (CÂMARA; BASTOS e VOLPE, 2012).

Associar o treinamento resistido, exercícios aeróbicos e alongamentos ao menos duas vezes na semana é imprescindível para a promoção da saúde e meIhora da capacidade física do idoso fragilizado (CÂMARA; BASTOS e VOLPE, 2012).

O aumento da expectativa da vida é um grande avanço para a humanidade, porém, manter o bem-estar durante o processo de envelhecimento é um grande desafio da saúde hoje, por isso, conhecer o perfil de QV de idosos frágeis é imprescindível, pois auxilia no planejamento dos cuidados de saúde destinados a estes indivíduos (ANDRADE et al., 2015).

As síndromes geriátricas no geral, em especial a fragilidade, necessitam de direcionamento e aprimoramento das políticas de atenção à saúde dos idosos, visando o bem-estar dos indivíduos e minimizar ou dificultar o agravamento e a evolução de tais déficits, evitando assim, maiores graves consequências (REIS et al., 2014).

\section{CONCLUSÃO}

Entre as medidas que podem ser realizadas para com o idoso frágil estão: a ação de uma equipe mul- tidisciplinar, que pode atuar visando a prevenção e a recuperação do paciente de forma global; a realização do diagnóstico precoce, permitindo a intervenção eficaz conforme as necessidades de cada indivíduo; utilização de ferramentas que permitam de forma rápida e simples a identificação do risco e/ou de fragilidade e a realização de atividades físicas, que proporcionam ao paciente, além dos benefícios físicos, autonomia e funcionalidade melhorando a qualidade de vida do idoso.

\section{REFERÊNCIAS}

ANDRADE, A.N.; FERNANDES, M.G.M.; NÓBREGA, M.M.L. et al. Análise do conceito fragilidade em idosos. Texto contexto-enferm, v. 21, n.4, p.748-756, 2012

ANDRADE, F.R.; MESQUITA, R.C.; CAMPOLINA, A.G. et al. Quality of life evaluation of frail elderly in Campinas, São Paulo. Rev. Assoc. Med. Bras, v.61, n.5, p.423-430, 2015.

ANDRADE, V.S.; PEREIRA, L.S.M. Influência da tecnologia assistiva no desempenho funcional e na qualidade de vida de idosos comunitários frágeis: uma revisão bibliográfica. Rev. Bras. Geriatr. Gerontol, v.12, n.1, p.113-122, 2009.

BRITO, K.Q.D.; MENEZES, T.N.; OLINDA, R.A. Incapacidade funcional e fatores socioeconômicos e demográficos associados em idosos. Rev. Bras. Enferm, v.68, n.4, p.633-640, 2015.

CADORE, E.L.; MONEO, A.B.B.; MENSAT, M.M. et al. Positive effects of resistance training in frail elderly patients with dementia after long-term physical restraint. Age, v.36, n.2, p.801-811, 2014.

CADORE, E.L.; CASAS-HERRERO, A.; ZAMBOM-FERRARESI, F. et al Multicomponent exercises including muscle power training enhance muscle mass, power output, and functional outcomes in institutionalized frail nonagenarians. Age, v.36, n.2, p. 773-785, 2014.

CADORE, E.L.; RODRÍGUEZ-MAÑAS, L.; SINCLAIR, A. et al. Effects of Different Exercise Interventions on Risk of Falls, Gait Ability, and Balance in Physically Frail Older Adults: A Systematic Review. Rejuvenation Research, v.16, n.2, p.105-114, 2013.

CÂMARA, L.C.; BASTOS, C.C.; VOLPE, E.F.T. Exercício resistido em idosos frágeis: uma revisão da literatura. Fisioter. Mov, v.25, n.2, p.435-443, 2012.

CORDEIRO, L.M.; PAULINO, J.L.; BESSA, M.E.P. et al. Qualidade de vida do idoso fragilizado e institucionalizado. Acta paul. Enferm, v.28, n.4, p.361-366, 2015. 
COSTA, T.B.; NERI, A.L. Medidas de atividade física e fragilidade em idosos: dados do FIBRA Campinas, São Paulo, Brasil. Cad. Saúde Pública, v.27, n.8, p.1537-1550, 2011.

FAIRHALL, N.; SHERRINGTON, C.; KURRLE, S.E. et al. Effect of a multifactorial interdisciplinary intervention on mobility-related disability in frail older people: randomised controlled trial. BMC Medicine, v.10, n.120, 2012.

FERNANDES, M.G.M.; ANDRADE, N.A.; NÓBREGA, L.M.M. Antecedents of frailty in the elderly: a systematic revision. Online Brazilian Journal of Nursing, v.9, n.1, 2010.

GERAEDTS, H.A.; ZIJLSTRA, W.; ZHANG, W. et al. Bulstra S, Stevens $M$. Adherence to and effectiveness of an individually tailored home-based exercise program for frail older adults, driven by mobility monitoring: design of a prospective cohort study. BMC Public. Health, v.14, n.570, 2014.

GONÇALVES, L.H.T.; ALVAREZ, A.M.; SENA, E.L.S. et al.Perfil da família cuidadora de idoso doente/fragilizado do contexto sociocultural de Florianópolis. Texto contexto - enferm, v.15, n.4, p.570-577, 2006.

LANA, L.D.; SCHNEIDER, R.H. Síndrome de fragilidade no idoso: uma revisão narrativa. Rev. bras. geriatr. Geronto, v.17, n.3, p. 673-680, 2014.

LENARDT, M.H.; SOUSA, J.R.; CARNEIRO, N.H.K. et al. Atividade física de idosos e fatores associados à pré-fragilidade. Acta paul. Enferm, v.26, n.3, p.269-275, 2013.

LUSTOSA, L.P.; SILVA, J.P.; COELHO, F.M.M. et al. Efeito de um programa de resistência muscular na capacidade funcional e na força muscular dos extensores do joelho em idosas pré-frágeis da comunidade: ensaio clínico aleatorizado do tipo crossover. Rev. bras. Fisioter, v.15, n.4, p.318324, 2011.

MCDERMID, R.C.; STELFOX, H.T.; BAGSHAW, S.M. Frailty in the critically ill: a novel concept. Critical Care. v.15, n.1, p.301, 2011.

MORETTO, M.C.; MATERA, R.A.A.; LIBERALESSO, A.N. et al. Relação entre estado nutricional e fragilidade em idosos brasileiros. Rev Bras Clin Med, v.10, n.4, p.267-71, 2012.
MORLEY, J.E.; VELLAS, B.; KAN, A.V. et al. "Frailty Consensus: A Call to Action." J. Am. Med. Dir. Assoc, v.14, n.6, p.392397,2013

OLIVEIRA, D.R.; BETTINELLI, L.A.; PASQUALOTTI, A. et al. Prevalência de síndrome da fragilidade em idosos de uma instituição hospitalar. Rev. Latino-Am. Enfermagem, v.21, n.4, p. $891-898,2013$

PEGORARI, M.S.; TAVARES, D.M.S. Fatores associados à síndrome de fragilidade em idosos residentes em área urbana. Rev. Latino-Am. Enfermagem, v.22, n.5, p.874-882, 2014.

REMOR, C.B.; BÓS, A.J.G.; WERLANG, M.C. Características relacionadas ao perfil de fragilidade no idoso. Sci. med, v..21, n.3, 2011.

REIS, J.W.M.; CARNEIRO, J.A.O.; COQUEIRO, R.S. et al. Préfragilidade e fragilidade de idosos residentes em município com baixo Índice de Desenvolvimento Humano. Rev. Latino-Am. Enferm, v.22, n.4, p.654-661, 2014

STORTI, L.B.; FABRÍCIO-WHEBE, S.C.C.; KUSUMOTA, L. et al. Fragilidade de idosos internados na clínica médica da unidade de emergência de um hospital geral terciário. Texto contexto - enfer, v.22, n.2, p.452-459, 2013.

TRIBESS, S.; VIRTUOSO, J.J.S.; OLIVEIRA, R.J. Atividade física como preditor da ausência de fragilidade em idosos. Rev Assoc. Med. Bras, v.58, n.3, p.341-347, 2012.

VALENÇA, T.D.C.; SILVA, L.W.S. Fisioterapia no Cuidado ao Sistema Familiar: Uma Abordagem à Pessoa Idosa Fragilizada. Rev. Tem. Kairós Gerontol, v.14, n.3, p.89-104, 2011.

VILLAREAL, D.T.; SMITH, G.I.; SINACORE, D.R. et al. Regular multi-component exercise increases physical fitness and muscle protein anabolism in frail, obese, older adults. Obesity. Silver Spring, Md, v.19, n.2, p.312-318, 2011.

ZAK, M.; SWINE, C.; GRODZICKI, T. Combined effects of functionally-oriented exercise regimens and nutritional supplementation on both the institutionalised and free-living frail elderly (double-blind, randomised clinical trial). BMC Public. Health, v.9, n.39, 2009. 